\title{
Epidemiology of dengue: past, present and future prospects
}

This article was published in the following Dove Press journal:

Clinical Epidemiology

19 August 2013

Number of times this article has been viewed

\author{
Natasha Evelyn Anne \\ Murray ${ }^{1,2}$ \\ Mikkel B Quam' \\ Annelies Wilder-Smith ${ }^{1,3}$ \\ 'Institute of Public Health, University \\ of Heidelberg, Heidelberg, Germany; \\ ${ }^{2}$ Population Health, Waikato District \\ Health Board, Hamilton, New Zealand; \\ ${ }^{3}$ Lee Kong Chian School of Medicine, \\ Nanyang Technological University, \\ Singapore
}

\begin{abstract}
Dengue is currently regarded globally as the most important mosquito-borne viral disease. A history of symptoms compatible with dengue can be traced back to the Chin Dynasty of 265-420 AD. The virus and its vectors have now become widely distributed throughout tropical and subtropical regions of the world, particularly over the last half-century. Significant geographic expansion has been coupled with rapid increases in incident cases, epidemics, and hyperendemicity, leading to the more severe forms of dengue. Transmission of dengue is now present in every World Health Organization (WHO) region of the world and more than 125 countries are known to be dengue endemic. The true impact of dengue globally is difficult to ascertain due to factors such as inadequate disease surveillance, misdiagnosis, and low levels of reporting. Currently available data likely grossly underestimates the social, economic, and disease burden. Estimates of the global incidence of dengue infections per year have ranged between 50 million and 200 million; however, recent estimates using cartographic approaches suggest this number is closer to almost 400 million. The expansion of dengue is expected to increase due to factors such as the modern dynamics of climate change, globalization, travel, trade, socioeconomics, settlement and also viral evolution. No vaccine or specific antiviral therapy currently exists to address the growing threat of dengue. Prompt case detection and appropriate clinical management can reduce the mortality from severe dengue. Effective vector control is the mainstay of dengue prevention and control. Surveillance and improved reporting of dengue cases is also essential to gauge the true global situation as indicated in the objectives of the WHO Global Strategy for Dengue Prevention and Control, 2012-2020. More accurate data will inform the prioritization of research, health policy, and financial resources toward reducing this poorly controlled disease. The objective of this paper is to review historical and current epidemiology of dengue worldwide and, additionally, reflect on some potential reasons for expansion of dengue into the future.
\end{abstract}

Keywords: evolution, geographic expansion, travel, climate change

\section{Introduction}

Dengue is an acute mosquito-borne viral infection that places a significant socioeconomic and disease burden on many tropical and subtropical regions of the world. ${ }^{1,2}$ It is currently regarded as the most important arboviral disease internationally as over $50 \%$ of the world's population live in areas where they are at risk of the disease, and approximately $50 \%$ live in dengue endemic countries. ${ }^{2-6}$

Correspondence: Professor A WilderSmith, Institute of Public Health, Im Neuenheimer Feld 365, University of Heidelberg, 69I20 Heidelberg, Germany Tel +49622I564864

Email anneliesws@gmail.com

\section{Dengue virus}

There are four distinct dengue virus serotypes, all of which originate from the family Flaviviridae and genus Flavivirus., ${ }^{3,7}$ The serotypes are termed DENV-1, DENV-2, 
DENV-3, and DENV-4, and infection with any of the four viruses results in lifelong immunity to that specific serotype. ${ }^{4,9,10}$ Each of the four serotypes has been individually found to be responsible for dengue epidemics and associated with more severe dengue..$^{8,11}$

\section{Dengue disease and clinical management}

Dengue is a complex disease with a wide spectrum of clinical presentations, which often goes unrecognized or is misdiagnosed as other fever-causing tropical diseases. ${ }^{12,13}$ Following the period of incubation, most patients experience a sudden onset of fever which can remain for 2-7 days and is often accompanied with symptoms such as myalgia, arthralgia, anorexia, sore throat, headaches, and a macular skin rash., ${ }^{74}$ It is during this period that differentiating dengue from other febrile diseases proves troublesome. ${ }^{15}$ The majority of people experience a self-limiting clinical course, which does not progress to the severe forms of dengue, dengue hemorrhagic fever (DHF), or dengue shock syndrome (DSS). Secondary dengue infections or particularly virulent viral strains are two factors thought to be associated with increased risk of severity. ${ }^{2,16}$ In severe cases, thrombocytopenia and increased vascular permeability can result in hemorrhagic and shock complications. Currently, neither a vaccine nor specific antiviral therapy exists. ${ }^{10,17,18}$ However, with prompt case detection and appropriate clinical management, including initiation of intravenous rehydration, the case fatality of severe dengue can be lower than $1 \% .{ }^{11}$ At present, due to a lack of available treatment, control of dengue focuses on effective vector control methods, which are limited. ${ }^{10,19,20}$

\section{Dengue vector and vector control}

The main arthropod vector for transmission of the dengue viruses is Aedes aegypti (A. aegypti). ${ }^{7}$ The second, less effective vector, Aedes albopictus (A. albopictus), feeds on multiple species of vertebrates, but has still been shown to be responsible for some dengue transmission. ${ }^{8}$ Significantly, the Aedes mosquitos are predominantly active during daylight hours, which poses difficulties in controlling the vector. ${ }^{7,21}$ A. aegypti mosquitoes are now extensively spread in both the tropics and subtropics. ${ }^{19}$ The mosquito is renowned for its efficient 'vectorial capacity' with a high affinity for human blood, high susceptibility to the four dengue virus serotypes, and being highly adapted to urban living. ${ }^{8,20,22,23} \mathrm{~A}$. aegypti mosquitoes breed in and around houses in regular water containers or disposed water-holding vessels. ${ }^{24}$ Due to this location of development and their limited flight range, female $A$. aegypti tend to persist in a domesticated environment. ${ }^{19}$ It is for this reason that humans are presumed the main cause of spread of dengue between communities. ${ }^{8}$ The wider prevention and control of dengue is currently reliant on vector control methods. These include environmental, biological, and chemical vector control management strategies and methodologies. ${ }^{19}$

The objectives of this paper are to review the current epidemiology of dengue worldwide as well as looking at its origins and history. Furthermore, we will attempt to elaborate on some of the reasons for the anticipated further expansion and increase of dengue in the future.

\section{Dengue in the past}

Dengue has been present for centuries. The first recorded symptoms compatible with dengue were noted in a Chinese medical encyclopedia in 992 AD, however originally published by the Chin Dynasty centuries earlier (265-420 AD), prior to being formally edited. ${ }^{25}$ The disease was referred to as 'water poison' and was associated with flying insects. ${ }^{26}$ Epidemics that resembled dengue, with similar disease course and spread, occurred as early as 1635 and 1699 in the West Indies and Central America, respectively. ${ }^{27} \mathrm{~A}$ major epidemic occurred in Philadelphia in 1780 and epidemics then became common in the USA into the early 20th century, the last outbreak occurring in 1945 in New Orleans..$^{23,27}$ The viral etiology and the transmission by mosquitoes were only finally determined in the 20th century.

The origin of the primary mosquito vector, A. aegypti, is debated to be from either Africa or Asia. ${ }^{8,23}$ Regardless, by 1800 it was widespread throughout urban tropical coastal cities of the world due to the use of shipping vessels with commercial expansion. ${ }^{8,23,28}$ These shipping vessels allowed transportation of breeding sites for the vector along with humans to complete the transmission cycle, allowing for slow but evident introduction of the virus and the mosquito to coastal destinations around the world. ${ }^{28}$ Epidemics were spaced by 10-40 year intervals due to this shipping mode of transport. ${ }^{23,25,28}$ Expansion of the disease heightened during World War II (WWII), when troops began to disperse inland and utilize modern transportation within and between countries; thus epidemic dengue became more far-reaching. ${ }^{23}$ By the end of the war, transportation and rapid urbanization led to increased transmission of dengue and hyperendemicity (multiple serotypes present) in most South East Asian countries, with subsequent emergence of the severe forms of dengue. ${ }^{2,22}$

Following WWII, dengue epidemics appeared to be under control in Central and South America. The elimination of $A$. aegypti, due to collaborative efforts with the yellow fever disease control campaign initiated by the Pan American 
Health Organization (PAHO), effectively restricted the transmission of dengue throughout the American continent. ${ }^{3,8,22,29}$ The lull in dengue epidemics in the Americas was short-lived, as the control campaign was discontinued in the 1970s. By the 1980s incidence had increased, and by 1995 pre-campaign levels were present in the Americas. ${ }^{22}$ Geographic expansion of epidemic dengue from South East Asia in the late 20th century saw regions in the Pacific and Americas escalate from being non-endemic with no dengue serotypes circulating, to hypoendemic (one serotype present), and some hyperendemic..$^{22,28}$

Global dengue incidence has increased precipitously over the last five decades and severe dengue cases have also expanded. ${ }^{2,46}$ Prior to 1970 , only nine countries had experienced severe dengue cases, a number which has since quadrupled..$^{30}$

\section{Current global situation of dengue}

Up to 3.6 billion people are estimated to now live in tropical and subtropical areas where the dengue viruses have the potential to be transmitted. ${ }^{2,431}$ Global estimates vary, but regularly approximate 50 million to 200 million dengue infections, 500,000 episodes of severe dengue (DHF/DSS), and over 20,000 dengue related deaths occur annually. ${ }^{3,29}$ Figure 1 shows the geographic distribution of dengue cases reported in 2011.

In 2012, dengue was once again classified by the World Health Organization (WHO) as the 'most important mosquito-borne viral disease in the world'6 due to significant geographic spread of the virus and its vector into previously unaffected areas and the subsequent costly burden of disease it brings. ${ }^{2,411,32}$ Disability adjusted life year (DALY) estimates differ, but a 2009 estimate of DALYs lost due to dengue globally was 700,000 per year. ${ }^{33,34}$ In most countries, the main burden of this morbidity and mortality lies with children. ${ }^{35-38}$ Diaz-Quijano and Waldman ${ }^{39}$ conducted an ecological study investigating the determinants of the dengue mortality burden. Length of recognized endemicity, rainfall, and population density were all shown to be associated with dengue mortality in Latin America and the Caribbean. ${ }^{39}$

A study which reviewed all nations in the Americas (with available data from the PAHO, 2000-2007) estimated an aggregate annual cost of dengue for the Americas at US $\$ 2.1$ billion. ${ }^{29}$ Approximately $60 \%$ of this cost related to indirect or 'productivity' losses, and the figure notably excluded prevention costs. ${ }^{29}$ A study of twelve countries in South East Asia (using available data from 2001-2010) showed an aggregate annual economic burden of US\$950 million amongst the studied nations, with approximately $52 \%$ of these costs coming from productivity loss. ${ }^{40}$ This figure again excluded necessary prevention and vector control costs. ${ }^{40}$

Due to poor disease surveillance, low level of reporting, low case fatality rate, difficulties in diagnosis, and inconsistent comparative analyses, the true incidence and impact of dengue is likely significantly higher than that which is currently reported. ${ }^{2,18,41}$ Thus, the true global burden of disease and associated economic impact is unknown. ${ }^{6}$ However, Brady et $\mathrm{al}^{42}$ have conducted the first of a series of steps in evidence consensus

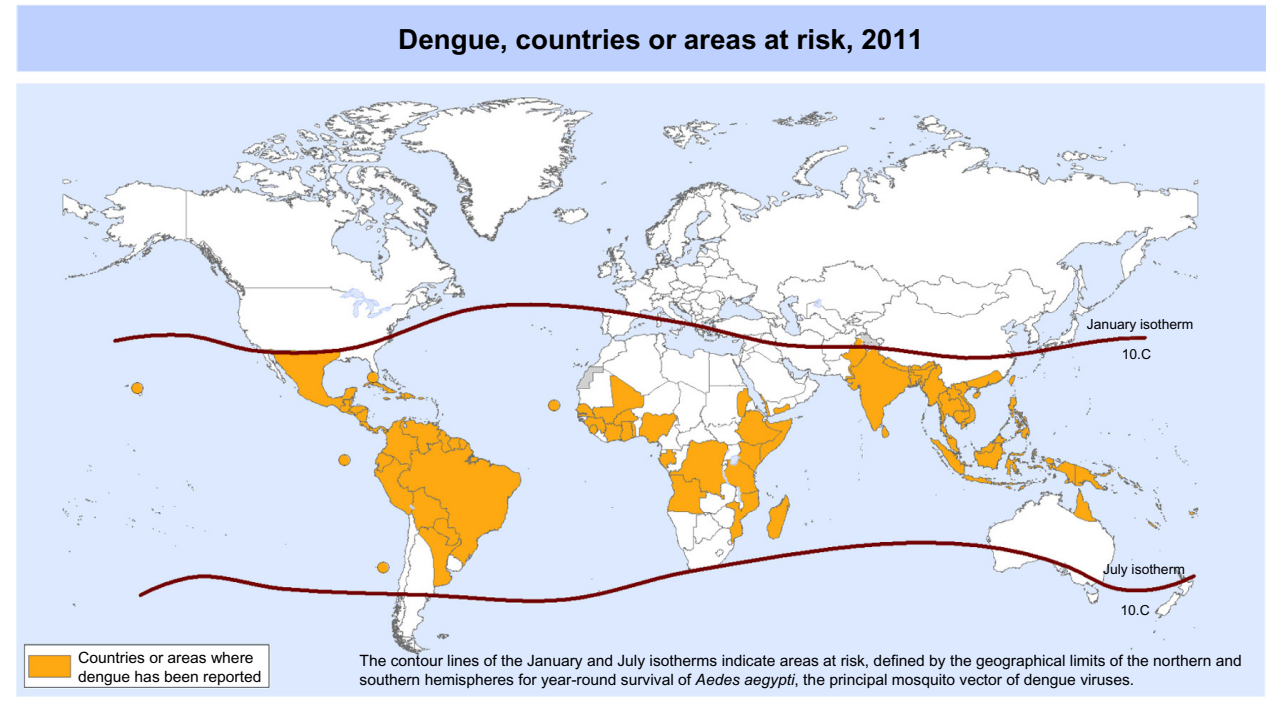

Figure I Countries or areas of the world where dengue was reported in 20II, as per data collected by the World Health Organization. ${ }^{32}$

Notes: Reprinted with permission from the World Health Organization Map Production: Public Health Information and Geographic Information Systems (GIS) World Health Organization. Dengue, countries or areas at risk, 20II. Geneva: World Health Organization (WHO). Available from: http://gamapserver.who.int/mapLibrary/Files/Maps/Global DengueTransmission_ITHRiskMap.png. Accessed July 30, 20I3. Copyright (C) $2012 .{ }^{32}$ The boundaries and names shown and the designations used on this map do not imply the expression of any opinion whatsoever on the part of the World Health Organization concerning the legal status of any country, territory, city or area or of its authorities, or concerning the delimitation of its frontiers or boundaries. Dotted and dashed lines on maps represent approximate border lines for which there may not yet be full agreement. 
mapping of global dengue incidence to better determine the population at risk. Their 2012 publication suggested an 'upper bound' total of 3.97 billion people living in 128 countries are at risk of dengue globally, 824 million in urban residences, and 763 in peri-urban residences. ${ }^{42}$ The same group published again in April 2013 using cartographic approaches. These data suggested 390 million dengue infections occur annually worldwide, including both apparent and inapparent infections, almost double the highest figure regularly reported to date. ${ }^{43}$

Despite the level of uncertainty on total numbers, we have evidence today that every WHO region now has dengue transmission and that there are more than 125 dengue endemic countries globally. ${ }^{5,31}$ Here we will review available data on dengue endemicity by WHO region.

\section{WHO Southeast Asia (SEA) region}

It is evident that dengue is now a worldwide concern; however, almost $75 \%$ of the global population exposed to dengue live in Asia-Pacific. ${ }^{6,8} 1.3$ billion of these at-risk individuals live in ten dengue endemic countries in SEA, and dengue is a leading cause of hospitalization and death in children from the region. ${ }^{8,28}$ The rates of disease reported in each of the SEA countries varies as they include either laboratory confirmed, probable, or suspected cases. ${ }^{40}$ However, it is clearly evident from data collated by WHO that, in SEA, an overall expansion of dengue has occurred over the last decade. ${ }^{8}$ In 2003 , eight countries in SEA had reported cases of dengue and, by 2009, all SEA member countries excluding the Democratic People's Republic of Korea reported indigenous cases. ${ }^{6,8}$ Epidemics continue to persist on regular 3-5 year cycles throughout SEA, and the number of reported cases continues to increase along with the severity of cases in many member countries. ${ }^{40} 187,333$ dengue cases were reported to WHO in 2010 from the region. ${ }^{31}$ Eight SEA countries are now also classified as hyperendemic with all four of the dengue virus serotypes present. ${ }^{8}$ Severe dengue is endemic in most SEA countries, with rates of severe dengue being 18 times higher in this region compared with the Americas. ${ }^{6,8,40}$

\section{WHOWestern Pacific region}

The WHO Western Pacific and SEA regions combined are attributed $75 \%$ of the global dengue disease burden. ${ }^{19,31}$ The number of reported cases of dengue has increased continuously over the past decade in the Western Pacific. ${ }^{44}$ 353,907 dengue cases and 1073 deaths were reported in the region as a whole in 2010. ${ }^{31,44}$

In the Asian subregion of the WHO Western Pacific region, the greatest burden of dengue currently originates from Cambodia, the Lao People's Democratic Republic, Malaysia, the Philippines, Singapore, and Vietnam. ${ }^{44}$ The number of reported cases increased in each of these nations over the past ten years, and all four serotypes have been identified in these high disease-burden settings. ${ }^{44}$ From the Pacific subregion, 91\% of reported cases came from French Polynesia, New Caledonia, Vanuatu, and Australia. ${ }^{44}$ Two dengue virus serotypes were reintroduced from the Americas to the Pacific Islands in 1964 (DENV-3) and in 1971 (DENV2), following a 25 year absence. ${ }^{2,27}$ The next decade saw gradual introduction of all four serotypes from Asia, which remain in circulation today. ${ }^{44}$ As a result, island nations in the Pacific show a particular susceptibility to dengue epidemics and severe dengue. ${ }^{6}$ In Australia, dengue activity, including indigenous outbreaks, occur in Northern Queensland where A. aegypti is present. ${ }^{10,44}$ In 2009 and 2010, more than 1000 cases of dengue were reported in Australia. ${ }^{44}$

\section{WHO region of the Americas}

Despite the absence of dengue transmission in the middle of the 20th century, almost all countries in the Americas now have hyperendemicity with indigenous dengue transmission. ${ }^{6}$ Epidemics occur cyclically in the region every three to five years, as they do in SEA, with increasing frequency and size, particularly in Latin America. ${ }^{19,29,31}$ In 2010, more than 1.6 million cases of dengue were reported in the Americas alone, 49,000 of these being severe dengue. ${ }^{5}$ Only two countries in Latin America remain to be without indigenous transmission, Uruguay and Continental Chile. ${ }^{45}$ Locally acquired cases of dengue have also now been reported in the USA. ${ }^{31}$ Due to the resurgence of dengue and its vector in the Americas over recent decades, PAHO has once again launched an initiative targeting vector control and prevention in the region. ${ }^{19}$ The 'Integrated Management Strategy for Dengue Prevention' is striving to reduce the disease and economic burden that dengue places currently on the Americas. ${ }^{6,45}$

\section{WHO African region}

Little has been known or reported about the situation in Africa amidst the geographic spread of dengue worldwide. ${ }^{6,13}$ Despite dengue not being officially reported to WHO by African countries and the probable under-recognition of dengue, evidence suggests that outbreaks are increasing in size and frequency in the region., ${ }^{3,6,12}$ Available outbreak data suggest 22 African countries reported sporadic cases or outbreaks between 1960 and 2010. ${ }^{6,13}$ Amarasinghe et al ${ }^{13}$ conducted a review of existing databases and literature in 2011 
that showed dengue transmission is endemic in 34 countries in the African region. 22 of these countries reported local disease transmission, 20 reported lab-confirmed cases, and two reported clinical cases alone. ${ }^{13,31}$ No 'local' reports of dengue occurred in the remaining 12 countries, only in travelers returning to non-endemic countries. ${ }^{13}$ All four dengue virus serotypes have been seen in Africa, however, DENV-2 appears to have caused most epidemics. ${ }^{13}$ Notably, in 2009, the Cape Verde archipelago had a large dengue outbreak involving more than 17,000 cases and linked to DENV-3. ${ }^{46,47}$ Due to the significant endemicity of malaria throughout the African region, the majority ( $>70 \%$ ) of 'febrile illnesses', including dengue, are likely to be misdiagnosed and treated as malaria. ${ }^{13}$ This negatively impacts attempts to draw a comprehensive picture of the epidemiology of dengue in the region and establish regular surveillance, outbreak monitoring, and relevant prevention and control activities. ${ }^{6,13}$

\section{WHO European region}

The last reported epidemic of dengue in Europe was between 1926 and 1928 in Greece. ${ }^{6}$ This epidemic implicated A. aegypti as the predominant vector and saw high mortality of cases. No dengue transmission had been reported since this time until A. albopictus became established in Europe in the 1990s as a result of increasing global trade of used tires. ${ }^{5,6,30}$ Today, there is a very real and apparent threat of dengue outbreaks in Europe. Imported cases in travelers are seen frequently and, in 2010, local transmission of dengue was reported in both Croatia and France., 5 The Madeira Islands of Portugal have been in the midst of an outbreak since October 2012. This outbreak had resulted in 2164 cases by February 2013, with 78 imported cases from recent travelers to Madeira detected in 13 other countries throughout Europe..$^{5,48,49}$ Thus, despite Europe being free of dengue for the majority of the 20th century, the global expansion of dengue is finally impacting the region.

\section{WHO Eastern Mediterranean region}

In the Eastern Mediterranean region, dengue is classified as an 'emerging disease'. ${ }^{6}$ Cases have only been officially reported to WHO for the last 2 decades, during which time three countries - Saudi Arabia, Pakistan, and Yemen - have had multiple outbreaks. ${ }^{6}$ For example, in 2011, the city of Lahore in Pakistan experienced a major dengue outbreak associated with 21,685 confirmed cases and 350 deaths, mainly due to DENV-2. ${ }^{50,51}$ Smaller outbreaks involving multiple serotypes of dengue virus are now being reported more frequently from countries such as Sudan, Djibouti, and Somalia. ${ }^{6}$ This highlights the probable geographic expansion of dengue within the Eastern Mediterranean Region, as with elsewhere globally.

\section{Dengue in the future}

Many experts hypothesize that dengue will increase in the future, including geographic expansion, incidence and reporting to WHO. ${ }^{10,23,52,53}$ It is therefore important to elaborate on some of the potential factors that drive dengue activity, as well as the global strategic direction to address this growth.

\section{Viral evolution}

Dengue viruses have been cataloged as having a low, medium, or high epidemiological impact according to their likelihood for human transmission and the clinical severity of dengue epidemics. ${ }^{54}$ In other words, some viruses largely prevail in sylvatic cycles among non-human primate populations, rarely transmitting to humans, while others are the viral agents causing mild dengue fever. ${ }^{54}$ Still other genotypes having higher virulence correlate with cases and epidemics characterized by more severe disease manifestations. ${ }^{54}$ For instance, there are some DENV-2 and DENV-3 genotypes found more commonly in the Americas which are known to be comparatively less virulent than Asian genotypes of the same serotype, as evidenced by reduced growth in both mosquitoes and culture. ${ }^{10,54}$ Wang et $\mathrm{al}^{55}$ demonstrated that domain III may play a role in viral adaptation to naïve hosts, whether mosquito or human, through analysis of modifications to the envelope protein postulated to correlate with endemic and/or epidemic emergence. ${ }^{55}$ Genotypes with greater virulence are driving out virus strains of lesser epidemiological impact, as evidenced by phylogenetic and epidemiological analyses..$^{23,54}$

\section{Climate change factors}

Temperature is known to play a role in adult vector survival, viral replication, and infective periods..$^{23,56-58}$ Increases of temperature may result in increased survival and or migration of vectors into previously non-endemic geographic areas outside the tropics. ${ }^{53}$ As the proliferation of Aedes mosquitoes is climate dependent, climate or meteorological factors can potentially provide useful information in predictive models. Weather variability has shown to be predictive of dengue activity. ${ }^{57-61}$ According to the Intergovernmental Panel on Climate Change, mean temperatures are predicted to rise globally. ${ }^{62}$ This may create climatic and environmental conditions conducive to the proliferation of Aedes species in areas that are currently non-endemic. The climatic suitability of many currently non-endemic areas and climatic similarity with endemic areas suggests that both $A$. aegypti 
and $A$. albopictus could become established or reestablished in the near future. ${ }^{63}$ A study conducted in the Southwest Pacific suggested that global temperature increases observed over the last four decades corresponded with increased risk of dengue outbreaks. ${ }^{64}$ Some studies on climate change and dengue show possible increase in transmission due to higher temperatures, humidity, and precipitation associated with changes in climate..$^{53,61,65}$ This supports the notion that observed climatic changes, including increased average global temperature and increased humidity, theoretically increase the epidemic potential of dengue. ${ }^{66,67}$ Based on longterm average vapor pressure prediction, climate change and population projections, Hales et $\mathrm{al}^{53}$ predicted that approximately $50 \%-60 \%$ of the global population would be living in areas at risk of dengue transmission by 2085 . If climate change did not occur as projected or was not taken into the model, only $35 \%$ would be at risk. ${ }^{53}$

The individual role climate change plays in the last decades' resurgence of dengue remains uncertain and is an area of current modeling research. ${ }^{67}$ Some authors also argue against climate as the main driver for dengue expansion. Beebe et $\mathrm{al}^{68}$ concluded from their Southeast Australian study that an increased risk of $A$. aegypti range expansion in Australia was due to the human adaptation of installing large domestic water storing containers as a response to persisting regional drying, rather than due to climate change itself. ${ }^{68}$ Furthermore, dengue and yellow fever caused multiple epidemics in the southern parts of the USA in the 18th, 19th, and early 20th centuries. Their eventual control was not due to a change in climate, but rather due to changes with industrialization and modernization. ${ }^{56,63}$

\section{Globalization, travel, and trade factors}

While climate change alone may not be a comprehensive and sufficient causal factor in the current and ongoing expansion of dengue, broader 'global change' may be. ${ }^{69}$ The 'global change' framework seeks to account for multiple factors of the modern world contributing to vector-borne communicable disease. ${ }^{69}$ Modern contributing factors to the rapid expansion of vector-borne communicable disease include globalization factors, such as travel and trade, associated with vector accommodating trends in modern human settlement and suitable climate conditions. ${ }^{2}$ The contributions of increased mobility, both of vector and human populations, may be the most important variable to explain the recent increase in dengue transmission. ${ }^{2}$

Climate and human settlement factors may enable and explain the risk of introduction or reintroduction of dengue into non-endemic zones where they border areas of endemic transmission. For other areas, further outside the tropics, the slight expansion due to climate change and human-vector interactions pale in comparison to factors of globalization. Globalization has been a main contributor and result of recent global economic development, creating a global ecosystem of exchange. ${ }^{2}$ The current global reality is one of international passenger travel and intercontinental exchange of goods. ${ }^{23} \mathrm{By}$ 2011, passenger air travel saw a 40-fold increase compared to the middle of the 20th century with ever increasing travel to and from dengue endemic areas. ${ }^{2}$ Human travel by those infected with dengue is thought to be the main driver of global transmission and expansion of the disease. ${ }^{2,66}$ Modern transport accounts for importation of dengue by overcoming natural barriers of travel time and geography, which had previously limited expansion from endemic areas into non-endemic areas. ${ }^{70}$ A recent model on the geospatial distribution of transmission via passenger air travel identified routes on which importation of dengue was an increased risk. ${ }^{70}$ Increased risk routes between the USA and Latin America, and also between Europe and Asia, were identified, ranked, and correlated with increased geographical distribution of the secondary dengue vector, A. albopictus. ${ }^{70}$ Intercontinental air travel between areas within the tropics has resulted in transmission of all four dengue virus serotypes in some areas. ${ }^{2,23}$ Overcrowded airports located in the tropics function as the ideal urban breeding ground and distribution point for dengue viruses within and outside current areas of transmission. ${ }^{2,70}$

As the global community trades and travels more and more, so too do communicable and vector-borne diseases. ${ }^{2}$ Further globalization factors, which are contributing to expansion of dengue transmission and risk of importation of dengue, include not only travel, but also trade. International transport of cargo and goods, especially via commercial sea shipment, can also export and import dengue's primary and secondary vectors, A. aegypti and A. albopictus, respectively. ${ }^{64}$ Transatlantic transport of used auto tires has been linked with the introduction of exotic American mosquito varieties into Italy, which contributed to other vector-borne disease epidemics. ${ }^{64,69,71}$ Given the vectors' suitability to breed and survive sea travel within water collected in a tire, their transport has contributed to a major public health threat in the last few decades, and this will only increase as more automobiles are consumed globally. ${ }^{72}$

\section{Settlement and socioeconomic factors}

Given the critique regarding climate as an independent factor in the observed expansion of dengue transmission, 
recent approaches seek to combine climate data with projected societal changes, including increased population and economic development in tropical and subtropical areas. ${ }^{52,57}$ While suitable climate factors are necessary to permit the resurgence and expansion of dengue transmission observed over the last 5 decades, human factors, including increasing global population, urbanization, and socioeconomic constraints on control measures, also contribute. Trends in current human settlement, together with rapidly expanded urban areas, exploding population density, and limited socioeconomic resources, suggest that the human factors, in addition to climate factors, may be necessary components in understanding current and future risks of dengue transmission. ${ }^{2,52}$ Settlement and socioeconomic factors combine with climatic suitably and globalized travel and trade to suggest that human populations and their collective actions strongly contribute to the transmission of dengue, in addition to mosquito vectors.

\section{Settlement factors}

Human factors, including both urban and rural settlement patterns, contribute to currently observed trends of increased incidence and expansion of dengue transmission. Rapid urbanization and population growth have been identified as strong contributing factors to the increase of global dengue transmission and geographic expansion. ${ }^{2,23}$ These two factors, particularly in low- and middle-income countries in tropical and subtropical regions, often precede the construction of necessary infrastructures for safe and comprehensive collection, storage, and disposal of water. ${ }^{69}$ Urban and suburban development may also provide new man-made breeding sites in the built environment, prior to human inhabitants occupying them. This has been shown even in well planned and financed situations such as the urban development endeavors in Putrajaya, Malaysia. ${ }^{73}$ In this manner, rapid urbanization facilitates the creation of urban breeding sites for the most potent dengue vector, A. aegypti. ${ }^{74}$ A. aegypti thrives in urban environments in that the mosquito breeds preferentially in the artificial containers often used in urban water collection. ${ }^{2}$ The increased density of both mosquito and human populations, as part of urban population growth, compounds this effect in terms both of vector suitability and transmission of dengue., ${ }^{2,75}$ While current research and policy interventions often treat dengue expansion as a phenomenon associated with urban human settlement, the incidence of the disease in rural areas is also on the rise. Some studies suggest that rural dengue incidence can even surpass urban and semi-urban communities within the same region. ${ }^{76,77}$ One such study in Cambodia found rural incidence to be 71/1000 person-seasons compared to urban incidence of $17 / 1000$ person-seasons during an outbreak of DENV-3 in $2007 .{ }^{77}$

Previous and ongoing underestimation of rural incidence may be attributable to similar vector-suitable breeding sites between some regions' urban-poor and rural communities. In addition, growing interconnectivity between rural and urban areas via increasing road infrastructure, combined with decreased access to diagnostics and surveillance may act as a silent conduit for rural dengue transmission and greater underestimation of rural incidence compared to more urban areas. ${ }^{77}$ The significant role and mechanisms of human involvement in creating a conducive ecology for dengue transmission, in addition to climate environmental factors, is being increasingly considered and modeled in current research. ${ }^{66,67}$

\section{Socioeconomic factors}

Historical dengue incidence and decline in Europe and the US, among other areas, suggests the role of socioeconomic development on dengue transmission and control. ${ }^{2,53,63}$ Multiple studies compared dengue endemicity and seroprevalence between neighboring border cities in Northern Mexico and Southern Texas. These highlight the importance of socioeconomic factors on the transmission of dengue, where climatic suitability was similar. ${ }^{78-81}$ In one such comparative cross-sectional study from 2004, current dengue seroprevalence was found to be $7.3 \%$ in Matamoros, Mexico, but only $2 \%$ in Brownsville, Texas, just across the border in the USA. ${ }^{78}$ Another similar serosurvey in 2005 suggested an even greater disparity between dengue incidence in Matamoros and Brownsville, reporting current dengue infection in $32 \%$ and $4 \%$, respectively, of the 273 study participants and estimating past dengue infection prevalence in $77 \%$ and $39 \%$, respectively. ${ }^{80}$ Key similarities observed among both cities included climate and geography, vector mosquito habitat and density, and human host social factors, for instance household size, use of insect screens, and basic sanitation. ${ }^{78-81}$ Socioeconomic and behavioral factors including income, water storage, usage of air-conditioning, waste disposal, and cross-border travel differed sustainably, as did dengue prevalence. ${ }^{78-81}$ In endemic areas, including the USA-Mexico border, more favorable socioeconomic factors resulting in higher utilization of air conditioning and domestic screening, as well as improved water and waste disposal infrastructure, are recommended to reduce larvae breeding sites and dengue transmission. ${ }^{78-81}$ Environmental management that aims to reduce, remove, and displace breeding 
sites from urban areas is recognized as a key mechanism to control dengue transmission. ${ }^{69}$

\section{Global strategic direction}

In light of the potential for continued expansion of dengue globally, it is essential to reflect on policy and strategic direction that attempts to reduce the impact of this disease. Dengue has been classified as a 'neglected topical disease,' based on the historical lack of coordinated efforts, political will, and research attention despite the significant disease, social, and economic burden it places internationally. ${ }^{6,82,83}$ This classification has encouraged prioritization of dengue via the WHO's Global Strategy for Dengue Prevention and Control, 2012-2020. ${ }^{6}$ The overall goal of this multi-sectoral strategy is 'to reduce the burden of dengue'. ${ }^{6}$ The document also defines objectives, technical elements, and enabling factors for effective implementation such as advocacy, partnership, coordination, and collaboration. The need to gain improved dengue disease burden estimates is one of three key objectives identified for dengue control with a timeframe for completion of 2015. More accurate epidemiological and surveillance data will enable further political prioritization for the currently 'neglected' disease. ${ }^{6}$ It would also enable improved decision making and rational allocation of financial, research, and other resources to the areas of greatest need. ${ }^{6,13}$ For example, epidemiological data will be essential in planning funding, allocation, and distribution of dengue vaccines that could potentially become available in the next decade. ${ }^{6,19}$

Sustainable vector control is one technical element of the Global Strategy for Dengue Prevention and Control, 2012-2020. ${ }^{6}$ In light of limited therapeutic strategies and the current lack of a vaccine, effective vector control methods are an essential component of the strategic direction to reduce dengue mortality and morbidity by $2020.6,19$ Integrated Vector Management (IVM) is the strategic approach promoted to countries by the WHO as a rational, cost-effective, and optimal decision-making process for vector control programs. ${ }^{6,84}$ For dengue vectors, this involves using a combination of approaches incorporating key elements of social mobilization, integration of chemical and non-chemical control methods targeting areas of high human-vector contact, evidence based decision-making guiding research and policy, as well as capacity building. ${ }^{84}$ Utilizing an effective integrated vector control strategy will aid in reduction of dengue transmission and the resulting disease burden.

Some researchers suggest dengue prioritization has now evolved and query how long it will be classified a 'neglected' disease. ${ }^{73,82}$ Furthermore, the impact of dengue has now progressed beyond those living in poverty as wealthier urbanized populations also have endemic dengue. ${ }^{6,73,82,85,86}$ Whilst this expands the experience and relevance of dengue to a broader group, the resulting impact on dengue control and future epidemiology is currently unknown. ${ }^{82}$ Irrespective, the poverty-promoting aspects of dengue, such as reduced economic potential with days off school and work, persist in the majority of dengue endemic settings. ${ }^{34}$ Perhaps, with continued expansion alongside improved epidemiological information, further prioritization and coordination of resources will be encouraged and we may see the objectives of the WHO 'Global Strategy' met by 2020.

\section{Conclusion}

Dengue is now endemic in more than 125 countries globally. ${ }^{5,31}$ Reasons for the currently observed and predicted expansion are multifactorial. They may include climate change, virus evolution, and societal factors such as rapid urbanization, population growth and development, socioeconomic factors, as well as global travel and trade. ${ }^{23,52}$

There is no antiviral therapy or vaccination available for dengue at this time, leaving only early detection and symptomatic treatment with fluid resuscitation essential for management of severe cases. ${ }^{19}$ As a result of limited therapeutic strategies, effective vector control methods are essential and are therefore promoted globally by the WHO through the strategic approach of IVM. ${ }^{6,84}$ For dengue, this approach targets the Aedes genus of mosquito in settings where high levels of human-vector contact occur. ${ }^{84}$ The WHO Global Strategy for Dengue Prevention and Control, 2012-2020, highlights the need for improved estimates of the true burden of dengue disease globally due to the currently presumed under-representation. ${ }^{6}$ Surveillance and reporting is paramount for effective dengue control, and more accurate quantification of the impact of dengue globally will allow improved political, financial, and research prioritization as well as informed decision making and enhanced modeling. ${ }^{2,18,40,43,87}$

The known social, economic, and disease burden of dengue internationally is alarming and it is evident that the wider impact of this disease is grossly underestimated..$^{2,29} \mathrm{An}$ international multi-sectoral response, such as that outlined in the WHO Global Strategy for Dengue Prevention and Control, 2012-2020, is now essential to reduce the significant influence this disease projects globally. ${ }^{6}$

\section{Disclosure}

The authors report no conflicts of interest in this work. 


\section{References}

1. Guzman MG, Kouri G. Dengue: an update. Lancet Infect Dis. 2002;2(1): $33-42$.

2. Gubler DJ. Dengue, Urbanization and Globalization: The Unholy Trinity of the 21(st) Century. Trop Med Health. 2011;39(Suppl 4):3-11.

3. Gubler DJ. The global emergence/resurgence of arboviral diseases as public health problems. Arch Med Res. 2002;33(4):330-342.

4. WHO TDR Global Alert and Repsonse Dengue/Dengue Haemorrhagic Fever [webpage on the Internet]. Geneva: World Health Organization (WHO); 2013 [cited March 3, 2013]. Available from: http://www.who. int/csr/disease/dengue/en/index.html.

5. Dengue and severe dengue: Fact Sheet No 117 [webpage on the Internet]. Geneva: World Health Organization (WHO); 2012 [cited March 4, 2013]. Available from: http://www.who.int/mediacentre/factsheets/ fs 117/en/index.html.

6. World Health Organization (WHO). Global Strategy for Dengue Prevention and Control, 2012-2020. Geneva: WHO Press; 2012.

7. International Travel and Health DENGUE [webpage on the Internet]. Geneva: World Health Organization (WHO); 2013 [cited March 5, 2013]; Available from: http:/www.who.int/ith/diseases/dengue/en/index.html.

8. WHO Regional Office for South-East Asia. Comprehensive Guidelines for Prevention and Control of Dengue and Dengue Haemorrhagic Fever, Revised and Expanded Edition. New Delhi: World Health Organisation South East Asia Regional Office; 2011.

9. Halstead SB. Etiologies of the experimental dengues of Siler and Simmons. Am J Trop Med Hyg. 1974;23(5):974-982.

10. Wilder-Smith A, Ooi EE, Vasudevan SG, Gubler DJ. Update on dengue: epidemiology, virus evolution, antiviral drugs, and vaccine development Curr Infect Dis Rep. 2010;12(3):157-164.

11. Gibbons RV, Vaughn DW. Dengue: an escalating problem. BMJ. 2002;324(7353):1563-1566.

12. Gubler DJ, Sather GE, Kuno G, Cabral JR. Dengue 3 virus transmission in Africa. Am J Trop Med Hyg. 1986;35(6):1280-1284.

13. Amarasinghe A, Kuritsk JN, Letson GW, Margolis HS. Dengue virus infection in Africa. Emerg Infect Dis. 2011;17(8):1349-1354.

14. Rigau-Perez JG, Clark GG, Gubler DJ, Reiter P, Sanders EJ, Vorndam AV. Dengue and dengue haemorrhagic fever. Lancet. 1998;352(9132):971-977.

15. Cao XT, Ngo NT, Wills B, et al. Evaluation of the World Health Organization standard tourniquet test and a modified tourniquet test in the diagnosis of dengue infection in Viet Nam. Trop Med Int Health. 2002; 7:125-132.

16. Endy TP, Anderson KB, Nisalak A, et al. Determinants of inapparent and symptomatic dengue infection in a prospective study of primary school children in Kamphaeng Phet, Thailand. PLoS Negl Trop Dis. 2011;5(3):e975.

17. Wilder-Smith A, Foo W, Earnest A, Sremulanathan S, Paton NI. Seroepidemiology of dengue in the adult population of Singapore. Trop Med Int Health. 2004;9(2):305-308.

18. Gubler DJ. The economic burden of dengue. Am J Trop Med Hyg. 2012;86(5):743-744

19. WHO. Dengue: Guidelines for Diagnosis, Treatment, Prevention and Control. New edition 2009. Geneva: World Health Organization; 2009.

20. Morrison AC, Zielinski-Gutierrez E, Scott TW, Rosenberg R. Defining challenges and proposing solutions for control of the virus vector Aedes aegypti. PLoS Med. 2008;5(3):e68.

21. Hawley WA. The biology of Aedes albopictus. JAm Mosq Control Assoc Suppl. 1988;1:1-39.

22. Gubler DJ, Clark GG. Dengue/dengue hemorrhagic fever: the emergence of a global health problem. Emerg Infect Dis. 1995;1(2): 55-57.

23. Wilder-Smith A, Gubler DJ. Geographic expansion of dengue: the impact of international travel. Med Clin N Am. 2008;92(6):1377-1390.

24. Mammen MP, Pimgate C, Koenraadt CJ, et al. Spatial and temporal clustering of dengue virus transmission in Thai villages. PLoS Med. 2008;5(11):e205.

25. Gubler DJ. Dengue/dengue haemorrhagic fever: history and current status. Novartis Found Symp. 2006;277:3-16.
26. Gubler D. Dengue and Dengue Hemorrhagic Fever. Clin Microbiol Rev. 1998;11(3):480-496.

27. Gubler DJ. Dengue and Dengue Hemorrhagic Fever: Its History and Resurgence as a Global Public Health Problem. In: Gubler DJ, Kuno G, editors. Dengue and Dengue Hemorrhagic Fever. London: CAB International; 1997:1-22.

28. Gubler DJ. Epidemic dengue/dengue hemorrhagic fever as a public health, social and economic problem in the 21 st century. Trend Microbiol. 2002;10(2):100-103.

29. Shepard DS, Coudeville L, Halasa YA, Zambrano B, Dayan GH. Economic impact of dengue illness in the Americas. Am J Trop Med Hyg. 2011;84(2):200-207.

30. Global Alert and Response - Impact of Dengue. Geneva: World Health Organization (WHO); 2013. Available from: http://www.who.int/csr/ disease/dengue/impact/en/.

31. Ferreira GL. Global dengue epidemiology trends. Rev Inst Med Trop Sao Paulo. 2012;54 (Suppl 18):S5-S6.

32. Dengue, countries or areas at risk, 2011. Geneva: World Health Organization (WHO); 2012. Available from: http://gamapserver.who.int/mapLibrary/ Files/Maps/Global_DengueTransmission_ITHRiskMap.png. Accessed July 30, 2013.

33. Cattand P, Guzmán MG, Jannin J, et al. Tropical Diseases Lacking Adequate Control Measures: Dengue, Leishmaniasis, and African Trypanosomiasis. In: Jamison DT, Breman JG, Measham AR, et al, editors. Disease Control Priorities in Developing Countries, 2nd ed. New York: Oxford University Press; 2006:451-466.

34. Hotez PJ, Fenwick A, Savioli L, Molyneux DH. Rescuing the bottom billion through control of neglected tropical diseases. Lancet. 2009;373:1570-1575.

35. Hammond SN, Balmaseda A, Perez L, et al. Differences in dengue severity in infants, children, and adults in a 3-year hospital-based study in Nicaragua. Am J Trop Med Hyg. 2005;73(6):1063-1070.

36. Egger JR, Coleman PG. Age and clinical dengue illness. Emerg Infect Dis. 2007;13(6):924-925.

37. Kittigul L, Pitakarnjanakul P, Sujirarat D, Siripanichgon K. The differences of clinical manifestations and laboratory findings in children and adults with dengue virus infection. J Clin Virol. 2007;39(2):76-81.

38. Guzman MG, Kouri G, Bravo J, Valdes L, Vazquez S, Halstead SB. Effect of age on outcome of secondary dengue 2 infections. Int J Infect Dis. 2002;6(2):118-124.

39. Díaz-Quijano FA, Waldman EA. Factors associated with dengue mortality in Latin America and the Caribbean, 1995-2009: an ecological study. Am J Trop Med Hyg. 2011;86:328-334.

40. Shepard DS, Undurraga EA, Halasa YA. Economic and disease burden of dengue in Southeast Asia. PLoS Negl Trop Dis. 2013;7(2):e2055.

41. Beatty ME, Beutels P, Meltzer MI, et al. Health economics of dengue: a systematic literature review and expert panel's assessment. Am JTrop Med Hyg. 2011;84(3):473-488.

42. Brady OJ, Gething PW, Bhatt S, et al. Refining the global spatial limits of dengue virus transmission by evidence-based consensus. PLoS Negl Trop Dis. 2012;6(8):e1760.

43. Bhatt S, Gething PW, Brady OJ, et al. The global distribution and burden of dengue. Nature. 2013;496:504-507.

44. Arima Y, Matsuiu T. Epidemiologic update of dengue in the western Pacific region, 2010. Western Pac Surveill Response J. 2011;2(2):1-5.

45. San Martin JL, Brathwaite O, Zambrano B, et al. The epidemiology of dengue in the Americas over the last three decades: a worrisome reality. Am J Trop Med Hyg. 2010;82(1):128-135.

46. Guzman MG, Halstead SB, Artsob H, et al. Dengue: a continuing global threat. Nat Rev Microbiol. 2010;8(Supp1 12):S7-S16.

47. Franco L, Di Caro A, Carletti F, et al. Recent expansion of dengue virus serotype 3 in West Africa. Euro Surveill. 2010;15(7).

48. Outbreak Notice-Dengue in Madeira, Portugal [webpage on the Internet]. Atlanta, GA: Centers for Disease Control and Prevention (CDC); 2013 [cited March 9, 2013]. Available from: http://wwwnc.cdc.gov/travel/ notices/outbreak-notice/dengue-madeira-portugal.htm. 
49. Epidemiological update: Outbreak of dengue in Madeira, Portugal [webpage on the Internet]. Stockholm: European Centre for Disease Prevention and Control (ECDC); 2013 [cited March 7, 2013]. Available from: http://www.ecdc.europa.eu/en/press/news/Lists/News/ECDC_ DispForm.aspx?List=32e43ee8-e230-4424-a783-85742124029a\&ID $=845 \&$ RootFolder $=\% 2$ Fen $\% 2$ Fpress $\% 2$ Fnews $\% 2$ FLists $\% 2$ FNews.

50. Rasheed SB, Butlin RK, Boots M. A review of dengue as an emerging disease in Pakistan. Public Health. 2013;127(1):11-17.

51. Khan MA, Ellis EM, Tissera HA, et al. Emergence and diversification of dengue 2 cosmopolitan genotype in Pakistan, 2011. PloS One. 2013; 8(3): 56391

52. Astrom C, Rocklov J, Hales S, Beguin A, Louis V, Sauerborn R. Potential distribution of dengue fever under scenarios of climate change and economic development. Ecohealth. 2012;9(4):448-454.

53. Hales S, de Wet N, Maindonald J, Woodward A. Potential effect of population and climate changes on global distribution of dengue fever: an empirical model. Lancet. 2002;360(9336):830-834.

54. Rico-Hesse R. Microevolution and virulence of dengue viruses. $A d v$ Virus Res. 2003;59:315-341.

55. Wang E, $\mathrm{Ni} \mathrm{H}, \mathrm{Xu} \mathrm{R}$, et al. Evolutionary relationships of endemic/epidemic and sylvatic dengue viruses. J Virol. 2000;74(7): 3227-3234.

56. Reiter P. Climate change and mosquito-borne disease. Environ Health Perspect. 2001;109 Suppl 1:141-161.

57. Gubler DJ, Reiter P, Ebi KL, Yap W, Nasci R, Patz JA. Climate variability and change in the United States: potential impacts on vector- and rodentborne diseases. Environ Health Perspect. 2001;109 Suppl 2:223-233.

58. Patz JA, Reisen WK. Immunology, climate change and vector-borne diseases. Trends Immunol. 2001;22(4):171-172.

59. Earnest A, Tan SB, Wilder-Smith A. Meteorological factors and El Nino Southern Oscillation are independently associated with dengue infections. Epidemiol Infect. 2012;140(7):1244-1251.

60. Wu PC, Guo HR, Lung SC, Lin CY, Su HJ. Weather as an effective predictor for occurrence of dengue fever in Taiwan. Acta Trop. 2007; 103(1):50-57.

61. Hii YL, Rocklov J, Ng N, Tang CS, Pang FY, Sauerborn R. Climate variability and increase in intensity and magnitude of dengue incidence in Singapore. Glob Health Action. 2009;2.

62. Contribution of Working Group I to the Fourth Assessment Report of the Intergovernmental Panel on Climate Change. Climate Change 2007: The Physical Science Basis. Cambridge, New York: Cambridge University Presss; 2007.

63. Reiter P. Yellow fever and dengue: a threat to Europe? Euro Surveill. 2010;15(10):19509

64. Banu S, Hu W, Hurst C, Tong S. Dengue transmission in the Asia-Pacific region: impact of climate change and socio-environmental factors. Trop Med Int Health. 2011;16(5):598-607.

65. Souza SS, Silva IG, Silva HH. Association between dengue incidence, rainfall and larval density of Aedes aegypti, in the State of Goiás. Rev Soc Bras Med Trop. 2010;43(2):152-155.

66. Russell RC, Currie BJ, Lindsay MD, Mackenzie JS, Ritchie SA, Whelan PI. Dengue and climate change in Australia: predictions for the future should incorporate knowledge from the past. Med $J$ Aust. 2009;190(5):265-268.

67. Van Kleef E, Bambrick H, Hales S. The geographic distribution of dengue fever and the potential influence of global climate change. TropIKA. net [serial on the Internet]. March 5, 2013. http://www.tropika.net/svc/ review/Chinnock-20110328-Review-Dengue-Climate.

68. Beebe NW, Cooper RD, Mottram P, Sweeney AW. Australia's dengue risk driven by human adaptation to climate change. PLoS Negl Trop Dis. 2009;3(5):e429.
69. Sutherst RW. Global change and human vulnerability to vector-borne diseases. Clin Microbiol Rev. 2004;17(1):136-173.

70. Gardner LM, Fajardo D, Waller ST, Wang O, Sarkar S. A predictive spatial model to quantify the risk of air-travel-associated dengue importation into the United States and europe. J Trop Med. 2012;2012:103679.

71. Napoli C, Salcuni P, Pompa MG, Declich S, Rizzo C. Estimated imported infections of Chikungunya and Dengue in Italy, 2008 to 2011. J Travel Med. 2012;19(5):294-297.

72. Reiter P. Aedes albopictus and the world trade in used tires, 1988-1995: the shape of things to come? J Am Mosq Control Assoc. 1998;14(1): 83-94.

73. Mulligan K, Elliott SJ, Schuster-Wallace C. The place of health and the health of place: dengue fever and urban governance in Putrajaya, Malaysia. Health Place. 2012;18(3):613-620.

74. Mendonca HF, Ferreira AL, Santos CB, et al. Breeding sites of Aedes aegypti in metropolitan vacant lots in Greater Vitoria, State of Espirito Santo, Brazil. Rev Soc Bras Med Trop. 2011;44(2):243-246.

75. Padmanabha H, Durham D, Correa F, Diuk-Wasser M, Galvani A. The interactive roles of Aedes aegypti super-production and human density in dengue transmission. PLoS Negl Trop Dis. 2012;6(8):e1799.

76. Reller ME, Bodinayake C, Nagahawatte A, et al. Unsuspected dengue and acute febrile illness in rural and semi-urban southern Sri Lanka. Emerg Infect Dis. 2012;18:256-263.

77. Vong S, Khieu V, Glass O, et al. Dengue incidence in urban and rural Cambodia: results from population-based active fever surveillance, 2006-2008. PLoS Negl Trop Dis. 2010;4:e903.

78. Brunkard JM, Robles Lopez JL, Ramirez J, et al. Dengue fever seroprevalence and risk factors, Texas-Mexico border, 2004. Emerg Infect Dis. 2007;13(10):1477-1483.

79. Brunkard JM, Cifuentes E, Rothenberg SJ. Assessing the roles of temperature, precipitation, and ENSO in dengue re-emergence on the TexasMexico border region. Salud Publica Mex. 2008;50(3):227-234.

80. Ramos MM, Mohammed H, Zielinski-Gutierrez E, et al. Epidemic dengue and dengue hemorrhagic fever at the Texas-Mexico border: results of a household-based seroepidemiologic survey, Dec 2005. Am J Trop Med Hyg. 2008;78(3):364-369.

81. Clark GG. Dengue and dengue hemorrhagic fever in northern Mexico and south Texas: do they really respect the border? Am JTrop Med Hyg. 2008;78(3):361-362.

82. Adams P. Dengue: neglected no more? TropIKA.net [serial on the Internet]. Jan 2011 [cited May 24, 2013]. http://www.tropika.net/svc/ review/Adams-20110110_Review_Dengue_2.

83. WHO TDR Neglected Tropical Disease Research [webpage on the Internet]. Geneva: World Health Organization (WHO); 2013 [cited May 24, 2013]. Available from: http://www.who.int/tdr/research/ntd/en/.

84. Dengue Control - Control Strategies [webpage on the Internet]. Geneva: World Health Organization (WHO); 2013 [cited March 2, 2013]. Available from: http://www.who.int/denguecontrol/control_strategies/en/.

85. Caprara A, Lima JW, Marinho AC, Calvasina PG, Landim LP, Sommerfeld J. Irregular water supply, household usage and dengue: a bio-social study in the Brazilian Northeast. Cad Saude Publica. 2009;25 Suppl 1:S125-S136.

86. Canyon DV. Historical analysis of the economic cost of dengue in Australia. J Vect Borne Dis. 2008;45:245-248.

87. Beatty ME, Stone A, Fitzsimons DW, et al. Best practices in dengue surveillance: a report from the Asia-Pacific and Americas Dengue Prevention Boards. PLoS Negl Trop Dis. 2010;4(11):e890. 


\section{Publish your work in this journal}

Clinical Epidemiology is an international, peer-reviewed, open access journal focusing on disease and drug epidemiology, identification of risk factors and screening procedures to develop optimal preventative initiatives and programs. Specific topics include: diagnosis, prognosis, treatment, screening, prevention, risk factor modification, systematic

Submit your manuscript here: http://www.dovepress.com/clinical-epidemiology-journal

\section{Dovepress}

reviews, risk \& safety of medical interventions, epidemiology \& biostatical methods, evaluation of guidelines, translational medicine, health policies \& economic evaluations. The manuscript management system is completely online and includes a very quick and fair peer-review system, which is all easy to use. 